\title{
Rendering Technique for Colored Paper Mosaic
}

\author{
Youngsup Park, Sanghyun Seo, YongJae Gi, Hanna Song, \\ and Kyunghyun Yoon \\ CG Lab., CS\&E, ChungAng University, \\ 221, HeokSuk-dong, DongJak-gu, Seoul, Korea \\ \{cookie, shseo, yj1023, comely1004, khyoon\}@cglab.cse.cau.ac.kr \\ http://cglab.cse.cau.ac.kr
}

\begin{abstract}
The work presented in this paper shows the way to generate colored paper mosaics using computer graphics techniques. Following two tasks need to be done to generate colored paper mosaic. The first one is to generate colored paper tile and the other one is to arrange the tile. Voronoi Diagram and Random Point Displacement have been used in this paper to come up with the shape of the tile. And, energy value that the tile has depending on its location is the factor to determine the best positioning of the tile. This paper focuses on representing the overlap among tiles, maintenance of the edge of input image, and various shapes of tiles in the final output image by solving two tasks mentioned above.
\end{abstract}

Keywords: Colored paper mosaic, Tile generation and Tile arrangement.

\section{Introduction}

Mosaic is an artwork formed by lots of small pieces called tile. It can be expressed in many different ways depending on the type and the position of tile. Photomosaics 1 shows the big image formed with small square tiles that are laid out on a grid pattern. Distinctive output was driven from the process of combining multiple images into one image. While Photomosaics shows the arrangement of tiles in a grid pattern, Simulated Decorative Mosaic 2 has tiles arranged in the direction of the edge of input image. This shows the similar pattern found in ancient Byzantine period. This pattern can also be found in Jigsaw Image Mosaics 3]. The only difference is to use various shape of image tiles instead of a single-colored square tiles. In this paper, we show especially how colored paper mosaic among various styles of mosaic artworks can be represented using computer graphics techniques.

To generate colored paper mosaic, following two issues need to be taken care. The first issue is to decide on the shape of colored paper tile and the second one is to arrange colored paper tile. Voronoi Diagram [9] and Random Fractal have been used in this paper to come up with the shape of colored paper tile. But the problem using Voronoi Diagram is that it makes the form of tile too plain since it generates only convex polygon. Therefore the method represented in this paper uses the predefined data of colored paper as database 
like Photomosaics. Then, it creates small piece of colored paper tile by clipping Voronoi polygon repetitively from the data of colored paper. Many different shapes of tiles like concave polygon can be expressed since it is made from repetitive tearing of one colored paper. And the energy value that colored paper tile has depending on its location is calculated to find the best positioning of colored paper tile. The location that has the biggest sum of energy value is defined as the best position. Tiles are placed at the point where the summation of energy value is the biggest by being moved and rotated toward the nearest edge.

\subsection{Related Work}

Existing mosaic studies focus on the selection, the generation, and the arrangement of tiles. We comparison the existing studies by classifying into two groups.

The studies of first group focus on the selection and the arrangement of tiles since they use fixed or predefined shapes of tiles. Photomasaics [1] creates image formed with various small pieces of image tiles. It is an algorithm that lays out selected image from the database in a grid pattern. It proposes an effective method of tile selection from database. But it is hard to keep the edge of image since the shape of tile in Photomosaic is all square ones. In the study of Simulated Decorative Mosaic[2], Hausner shows the similar pattern and techniques used in Byzantine era by positioning single-colored square tile in the direction of the edge of input image. It uses the methods of Centroidal Voronoi Diagram (CVD) and Edge Avoidance to arrange tiles densely. In Jigsaw Image Mosaics (JIM) 3], it shows extended technique by using arbitrary shapes of image tiles while Simulated Decorative Mosaic uses single-colored square tiles. It proposes solution of tile arrangement with Energy Minimization Framework.

The studies of second group propose the method only about the generation of tiles. Park [5] proposes passive colored paper mosaic generating technique that shape and arrangement of tiles is all done by the user's input. The proposed method uses Random Fractal technique for generating torn shaped colored paper tile. However, it gives the user too much works to do. To solve the problem passive technique has, automatic colored paper mosaic [6] using Voronoi Diagram is proposed. The majority works are done by computer and only part the user needs to do is to input a few parameters. It reduces heavy load of work on user side; however, it cannot maintain the edge of image since it arranges tiles without considering the edge. In order to solve this problem, another new technique [7] is suggested. In this new technique, it arranges Voronoi sites using Quad-Tree and clips the tiles according to the edge of image once it goes out of the edge. Even though this technique can keep the edge of images, it cannot express the real texture and shape of colored paper tearing since the polygon created using Voronoi Diagram becomes in a convex form and each polygon is not overlapped. Therefore, the existing study is not showing various shapes of tiles and the overlap among them. 


\section{Preprocessing}

\subsection{Data Structure of Colored Paper}

The data structure of colored paper is organized with 2 layers that contain the information such as the texture image and vertex shown as figure 1. The upper layer means visible space of colored paper that has the color value. And the lower layer means the white paper represented on the torn portion. To define the data structure of colored paper in advance gives two good aspects. The first one is that it can express various shape of colored paper tile like concave polygon besides convex one. This is because previously used colored paper is stored in the buffer and polygon clipping is repeated using Vonoroi Diagram as necessary. The other one is that different type of paper mosaic can be easily done by modifying data structure. If the image is used magazine, newspaper and so on instead of colored paper then it will be possible to come up with paper mosaic like Collage.
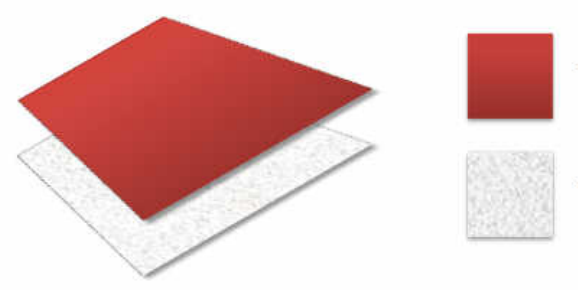

The color of colored paper (Layer 1)

The white paper (Layer 2)

Fig. 1. The data structure of colored paper object

\subsection{Image Segmentation}

At first, the necessary image processing works [1] like blurring are performed on the input image and the image is divided into several regions that have similar color in LUV space by using Mean-Shift image segmentation technique 8 . We call the region container. And the proposed mosaic algorithm is performed per container. However Mean-Shift segmentation can create small containers. If mosaic processing is performed in this stage, the colored paper tiles will not be attached to these small containers so it will result in lots of grout spaces in the result image as show in figure 4. Therefore, there is another step needed to integrate these small containers. To give flexibility and to show individual intention of expression, the process of integration of small containers is controlled by the user's input.

\section{The Generation of Colored Paper Tile}

\subsection{Determination of Size and Color}

To determine the size and the color of tile, the initial position where tile is attached is determined in advance by Hill-Climbing algorithm 4 . Hill-Climbing 
algorithm keeps changing the position till the function value converges to optimal point. Since normally big tiles are applied primarily from boundary rather than small ones in real life, the function is determined like equation 1 with following two factors: size and boundary. The size factor is defined by $D(x, y)$ that means the minimum distance value between pixel $(x, y)$ and boundary. And the boundary factor is defined by $D(x, y)-D(i, j)$ that means the sum of difference between neighbor pixels. Therefore, the position that has the largest value of $L(x, y)$ is regarded as an initial position.

$$
L(x, y)=\sum_{i=x-1}^{x+1} \sum_{j=y-1}^{y+1} D(x, y)-D(i, j) \cdot[x \neq i \& y \neq j]
$$

The size of colored paper tile is determined by distance from boundary. At first, we divide boundary pixels into two groups. The first group has smaller value than y of initial position, And the second group has larger value than y. Then, between two minimum distance values on each group, the small value set as the minimum size and the large value set as the maximum size.

Colored paper that has the similar color as the one in the initial position is selected. Colored paper is defined as single-colored. First of all, square area is built around the initial position for the size of tile and then the average value of RGB color in that area is selected.

\subsection{Determination of Shape}

There are two steps to determine the shape of colored paper tile. The first one is to determine the overall outline of tile to be clipped and the other one is to express torn effect. Voronoi Diagram is applied to decide the overall outline of tile. First, the area of colored paper is divided into several grids according to the size of tile to be torn. Then, Voronoi diagram is created by placing individual Voronoi site in each segment as shown in figure 2(b), The generated Voronoi diagram contains multiple Voronoi polygons so it needs to be decided to clip which polygon among them. Considering the fact that people start to clip from the boundary of the paper in real mosaic work, the polygon located near the boundary is decided to be torn first. Since there is always vertex in the boundary of colored paper as shown in figure $2(\mathrm{c})$, one of the polygons that contain vertex is randomly chosen. Once the outline of tile is determined by Voronoi polygon, it is necessary to apply torn effect to the boundary of determined outline. This torn effect is done by applying Random Point Displacement that is one of the Random Fractal techniques to colored paper's layer individually. Random Point Displacement algorithm is applied to the boundary of selected Voronoi polygons that is not overlapped with the boundary of colored paper. The irregularity of torn surface and white-colored portion can be expressed by perturbing the random point of edge continuously in the vertical direction. Lastly, clip the modified Voronoi polygon by Random Point Displacement algorithm as shown in figure $2(\mathrm{~d})$. 


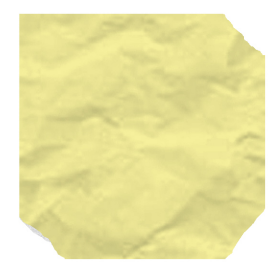

(a) Colored paper

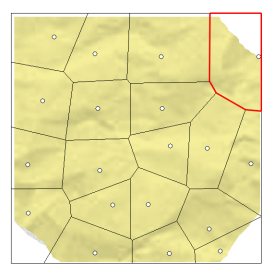

(b) Voronoi

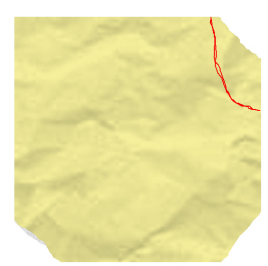

(c) Torn Effect

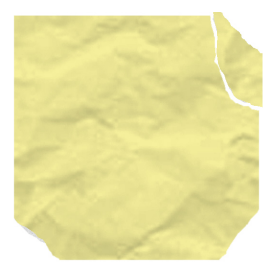

(d) Clipping

Fig. 2. The process of paper tearing

\section{The Arrangement of Colored Paper Tile}

There are two things to consider arranging colored paper tiles. First one is to maintain the edge of the input image and the other one is to get rid of empty spaces among tiles or between tile and the edge of the image. To maintain the edges of input image, the similar technique to Energy Minimization Framework of Jigsaw Image Mosaics is used in this paper. The energy function is defined first depending on the position of tile and the sum of it is calculated like $E(x, y)$ in equation 2 .

$$
\begin{gathered}
E(x, y)=P_{i}-P_{o}-P_{t} \\
\begin{cases}P_{i}=T_{\max } / 2-D(x, y) & \text { where }(x, y) \in C \text { and }(x, y) \notin T \\
P_{o}=W_{o} \cdot D(x, y) & \text { where }(x, y) \notin C \\
P_{t}=W_{t} \cdot D(x, y) & \text { where }(x, y) \in T\end{cases}
\end{gathered}
$$

$P_{i}, P_{o}, P_{t}$ shown in the expression above mean the number of pixels located in the inside of container, outside of container, and on the overlapped area with other tiles. And $W_{o}, W_{t}$ represent weight value depending on each location of pixel. The bigger the value of sum $E(x, y)$ is the better the position is to maintain the edges of input image. Therefore, the tile needs to be placed where the sum of $E(x, y)$ is the greatest. To get rid of empty spaces among tiles and between the tile and the edge of image, tile is moved and rotated into the direction of the nearest edge. This movement and rotation is continuously happening till the sum of $E(x, y)$ from equation 2 has convergence value or is not getting bigger

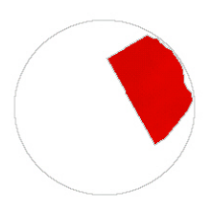

(a) The best case



(b) The worst case

(c) less overlapping

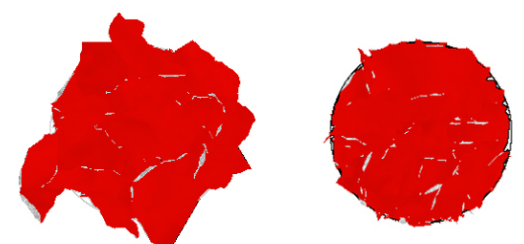

(d) edge keeping

Fig. 3. Positioning of colored paper tile 
any longer. The figure 3 shows four different situation of tile arrangement. The figure 3(b) shows the case that the tile is positioned outside of the edge of the image. Correction on tile's location needs to be done since it prevents the tile from keeping the edge of the image. Two tiles are overlapped too much in the figure $3(\mathrm{c})$ and it also needs to be modified. The figure $3(\mathrm{~d})$ shows the optimal arrangement of the tile. We can control this by adjusting the value of $W_{o}$ and $W_{t}$.

\section{Results}

The figure 4, 5 and 6 shows the result image rendered by assigning the size of tile of the source image between 4 and 100. The result shown in the figure 4 is the result of colored paper mosaic processed by only applying segmentation algorithm to the source image. The grout space appears where is segmented

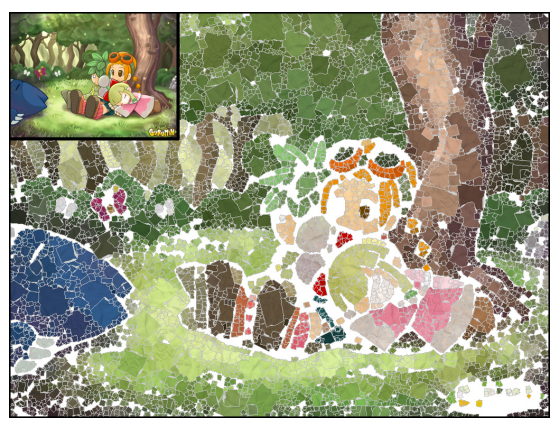

(a)

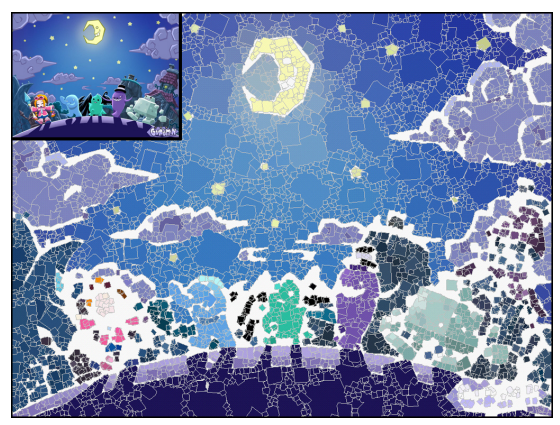

(b)

Fig. 4. The examples that have lots of grout spaces

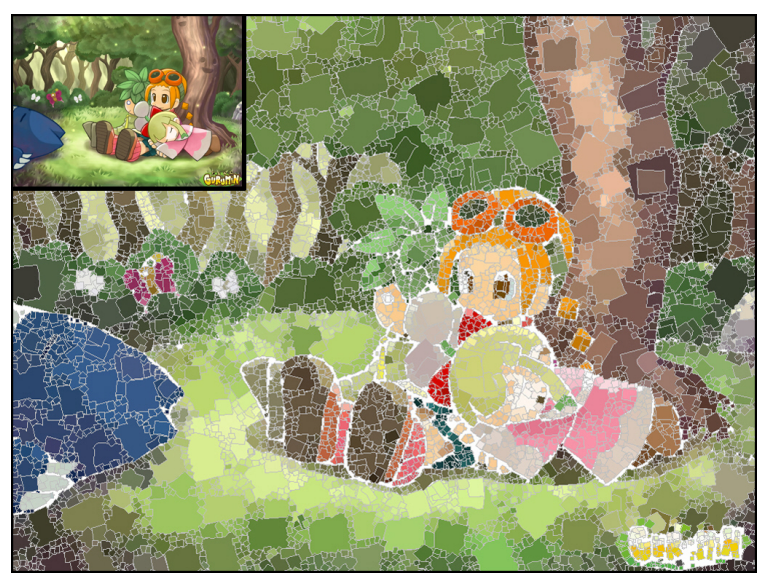

Fig. 5. The result of colored paper mosaic 




Fig. 6. The result of colored paper mosaic with height map

smaller than the size 4 since the minimum size of the tile is set to 4 . These smaller containers have to be integrated into the near container in order to get rid of grout spaces. The result of colored paper mosaic including container integration step is shown in the figure 5 . In the figure 5, the grout spaces shown in figure 4(a) are disappeared. Also, lots of small segments are removed by integration so the number of smaller size of tiles is reduced. And we can apply the texture effect to the result image by using texture mapping, height map [10], and alpha blending as shown in the figure 6. By adding these effects, the mosaic image gets more realistic.

\section{Discussion and Future Work}

The work presented in this paper shows the new method to generate colored paper tile with computer graphics techniques. The difference that this paper has is that it can maintain the edges of the input image and express the various shape of tile and overlaps among tiles. These three achievements are shown in the figure 4, 5] and 6.

The proposed method has some problems. First, too many small tiles are filled in between large tiles in the results. It is because grout spaces appear between the tile and the edge during the process of arranging the tile. It causes the problem to the quality of result image so it needs to be improved afterward. Therefore, another step to consider the edge of image during the tile generation is necessary. This additional step will reduce the generation of grout spaces among tiles or between the tile and the edge of image. Second, the performance of whole process is very low, since the tile arrangement is performed per pixel. Therefore it is needed to apply GPU or any other algorithms for improving the performance.

This paper also has some benefits like following. First, the proposed method can express the various shapes of tile and overlapping between other tiles. Second, 
if other types of paper like newspaper are used instead of colored paper then it will be possible to come up with another type of mosaic like Collage. It is easy to express other type of mosaic in computer graphics by modifying the data structure if more detailed and elaborate tile selection algorithm is applied.

\section{References}

1. Silver.R and Hawley.M (ed.): Photomosaics, New York: Henry Holt, 1997

2. Alejo Hausner : Simulating Decorative Mosaics, SIGGRAPH 2001, pp.573-580, 2001.

3. Junhwan Kim, Fabio Pellacini : Jigsaw Image Mosaics, SIGGRAPH 2002, pp. 657-664, 2002

4. Chris Allen : A Hillclimbing Approach to Image Mosaics, UW-L Journal of Undergraduate Research , 2004

5. Young-Sup Park, Sung-Ye Kim, Cheung-Woon Jho, Kyung-Hyun Yoon : Mosaic Techniques using color paper, Proceeding of KCGS Conference, pp.42-47, 2000

6. Sang-Hyun Seo, Young-Sup Park, Sung-Ye Kim, Kyung-Hyun Yoon : Colored Paper Mosaic Rendering, In SIGGRAPH 2001 Abstrac ts and Applications, p.156, 2001

7. Sang-Hyun Seo, Dae-Uk Kang, Young-Sup Park, Kyung-Hyun Yoon : Colored Paper Mosaic Rendering Based on Image Segmentation, Proceeding of KCGS Conference, pp27-34, 2001

8. D. Comanicu, P. Meer : Mean shift: A robust approach toward feature space analysis, IEEE Transaction on Pattern Analysis and Machine Intelligence, 24, 603-619, May 2002

9. Mark de Berg, M. V. Kerveld, M. Overmars and O. Schwarzkopf : Computational Geometry Algorithms and Applications, Springer, pp.145-161, 1997

10. Aaron Hertzmann : Fast Paint Texture, NPAR 2002, 2002

11. Rafael C. Gonzalez and Richard E. Woods : Digital Image Processing 2nd Edition, publish ed by Prentice Hall, 2002 